\title{
Microscopic description of fission dynamics: Toward a 3D computation of the time dependent GCM equation
}

\author{
D. Regnier ${ }^{1, a}$, N. Dubray ${ }^{1, b}$, N. Schunck ${ }^{2}$, and M. Verrière ${ }^{1}$ \\ 1 CEA, DAM, DIF, 91297 Arpajon, France \\ 2 Nuclear and Chemical Science Division, LLNL, Livermore, CA 94551, USA
}

\begin{abstract}
Accurate knowledge of fission fragment yields is an essential ingredient of numerous applications ranging from the formation of elements in the r-process to fuel cycle optimization in nuclear energy. The need for a predictive theory applicable where no data is available, together with the variety of potential applications, is an incentive to develop a fully microscopic approach to fission dynamics. One of the most promising theoretical frameworks is the time dependent generator coordinate method (TDGCM) applied under the Gaussian overlap approximation (GOA). However, the computational cost of this method makes it difficult to perform calculations with more than two collective degree of freedom. Meanwhile, it is well-known from both semi-phenomenological and fully microscopic approaches that at least four or five dimensions may play a role in the dynamics of fission. To overcome this limitation, we develop the code FELIX aiming to solve the TDGCM+GOA equation for an arbitrary number of collective variables. In this talk, we report the recent progress toward this enriched description of fission dynamics. We will briefly present the numerical methods adopted as well as the status of the latest version of FELIX. Finally, we will discuss fragments yields obtained within this approach for the low energy fission of major actinides.
\end{abstract}

\section{Introduction}

The growing need for nuclear data in applications such as the r-process study in astrophysics is an incentive to determine the fission yields for a wide range of systems, observables, and input channels. Since the 90 s, experimental efforts on inverse kinematics reactions fall in with this trend by providing extremely accurate yields data for various fissioning nuclei [1-4]. On the other side, building a predictive theory of fission applicable where no data is available remains a major challenge of nuclear physics. Over the last decades, part of the theoretical effort has been focused on predicting induced fission yields based on the time-dependent generator coordinate method (TDGCM) associated with the Gaussian overlap approximation (GOA) [5,6]. This formalism provides a fully quantum mechanical description of the time evolution of large collective motions in nuclei. Its application to the description of the fission dynamics has made continuous progress since 2005 [7-10]. However, because of the computational resources required by this approach, fission dynamics was only studied in 2-dimensional collective spaces. Yet, it is well-known from both semiphenomenological and fully microscopic approaches that at least four or five collective variables play a role in the dynamics of fission [11-14]. These reasons were the primary motivation to undertake TDGCM+GOA calculations within a 3-dimensional collective space.

a e-mail: david.regnier@cea.fr

b e-mail: noel.dubray@cea.fr
In this work, we give a quick overview of the TDGCM+GOA method applied to the prediction of fission yields. We briefly outline the fission yields of ${ }^{239} \mathrm{Pu}(\mathrm{n}, \mathrm{f})$ recently obtained within a 2-dimensional collective space. Finally, we emphasize our progress toward a 3-dimensional description of the fission dynamics.

\section{The TDGCM+GOA approach to compute fission yields}

The time-dependent generator coordinate method (TDGCM) provides a fully quantum mechanical description of the time evolution of the fissioning system. It starts from the general observation that the time evolution of the many-body fissioning system is governed by the timedependent Schrödinger equation. In our applications, the nuclear Hamiltonian $\hat{H}$ involved contains an effective twobody potential such as, e.g., the Skyrme or the Gogny interaction. Inserting the so called GCM ansatz [6] as well as the Gaussian overlap approximation (GOA) in a variational form of the time-dependent Schrödinger equation yields a time-dependent differential equation for the evolution of the system:

$i \hbar \frac{\partial}{\partial t} g(\boldsymbol{q}, t)=\left[-\frac{\hbar^{2}}{2} \sum_{k l} \frac{\partial}{\partial q_{k}} B_{k l}(\boldsymbol{q}) \frac{\partial}{\partial q_{l}}+V(\boldsymbol{q})\right] g(\boldsymbol{q}, t)$,

where

- The vector $\boldsymbol{q}$ contains the collective coordinates chosen to described the dynamics. They are in this work expectation values of the multipole moment 
operators $\hat{Q}_{\lambda \mu}$. The quadrupole moment labeled $q_{20}$ characterizes the elongation of the nucleus whereas the octupole moment $\left(q_{30}\right)$ is related to the asymmetry between the pre-fragments. The possible additional constraints $q_{40}$ is closely correlated to the size of the neck around scission.

- The unknown complex function $g(\boldsymbol{q}, t)$ is related to the state of the many body wave function of the compound system. It contains all the information about the dynamics. In the limit of sharply peaked norm kernels, the quantity $|g(\boldsymbol{q}, t)|^{2}$ can be interpreted as the probability density for the system to have the collective characteristics $\boldsymbol{q}$ at time $t$;

- The potential field $V(\boldsymbol{q})$ and the inertia tensor $B_{k l}(\boldsymbol{q})$ are fully determined by the knowledge of the effective Hamiltonian $\hat{H}$ and the collective coordinates.

The Eq. (1) is referred to as the TDGCM+GOA equation.

Our microscopic approach to compute the fission yields is a three-step process. First, we compute the potential and inertia fields for a range of values of the collective variables. This is the most time-consuming part, since any realistic two-dimensional fission calculation involves of the order of 50,000 points of the collective space. A second step consists in numerically solving the TDGCM+GOA Eq. (1) starting from an initial function $g(\boldsymbol{q}, t=0)$. This initial condition is arbitrarily built as a wave packet localized at low deformation and having an energy $1 \mathrm{MeV}$ above the inner potential barrier. In the third and final step, we extract fission product yields from the time-dependent solution of the dynamic equation by defining a frontier in the collective space. This frontier separates a domain mainly composed of scissioned configurations from configurations where the system is still a whole nucleus. Computing the probability for the system to cross through any point of this line provides a first estimate of the fission yields. This raw data is then convoluted with a Gaussian to take into account effects such as quantum fluctuations of particle number. A detailed description of the whole procedure can be found in [10].

\section{Prediction of actinide fission yields based on two collective variables}

The state of the art application of TDGCM+GOA to the problematic of fission relies on a 2-dimensional collective space. Recently, we performed a comprehensive investigation of the low energy ${ }^{239} \mathrm{Pu}(\mathrm{n}, \mathrm{f})$ reaction based on the collective space spanned by the expectation values of the quadrupole and octupole moments. The Fig. 1 emphasizes the pre-neutron fragments mass yields obtained in this framework for the two effective interactions SkM* [17] and D1S [18, 19]. The asymmetric nature of the ${ }^{240} \mathrm{Pu}$ fission is well described by the TDGCM approach with both density functionals. Part of this agreement comes from the fact that both calculations and experimental data are convolutions of a raw data set with a smoothing function: The Schillebeeckx et al. [15] data comes from a $2 \mathrm{E}$ measurement with a mass resolution of typically 4-5 mass units (FWHM) [20]. A measurement of both energy and velocity of the two fragments as the one performed by Nishio et al. [16] is also expected to provide a mass resolution significantly

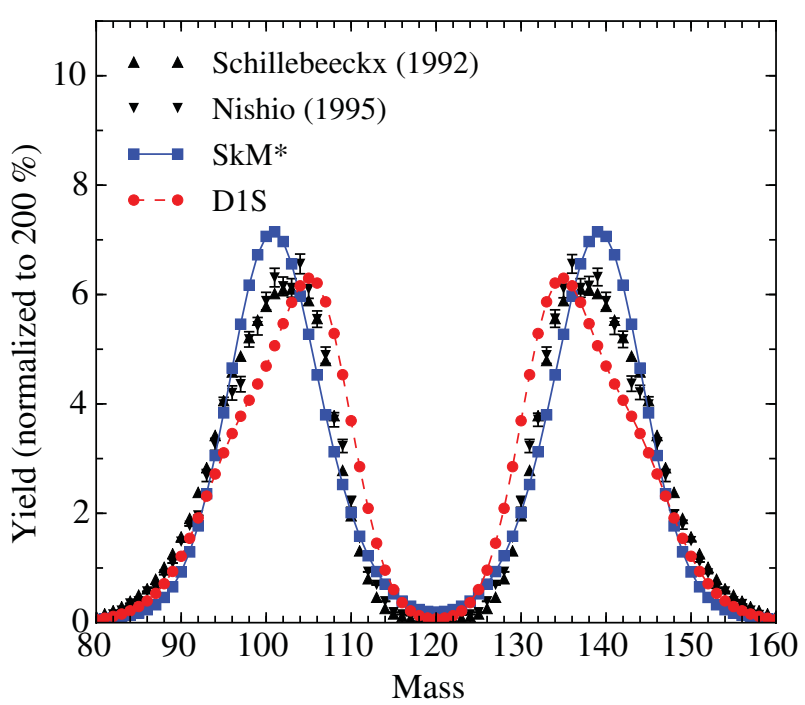

Figure 1. Pre-neutron mass yields for ${ }^{239} \mathrm{Pu}(\mathrm{n}, \mathrm{f})$. The $\mathrm{SkM}^{*}$ and D1S calculations are compared with two experimental datasets $[15,16]$. Figure taken from [10].

larger than one mass unit. Finally, the TDGCM dataset itself results from the convolution of a raw probability flux with a Gaussian of width $\sigma=4$ mass units. The centroids of the peaks agree within 2 mass units between theory and experiment. The SkM* EDF favors slightly more asymmetric configurations whereas we observe the opposite for D1S. Such a shift of the D1S peak toward symmetry was previously observed in work by Younes and Gogny relying on a different collective space and a totally different numerical implementation both of the HFB and TDGCM+GOA solver [8]. At this stage, no attempt has yet been made to understand if a change in one specific term of the EDF between $\mathrm{SkM}^{*}$ and D1S could explain the shift in the peak position. A sensitivity study of the mass yields with respect to the few parameters of our approach (e.g. initial state, collective inertia) was performed. Its results along with complementary calculations on ${ }^{236} \mathrm{U}(\mathrm{n}, \mathrm{f})$ show that our approach produces a robust qualitative description of the asymmetric fission in ${ }^{236} \mathrm{U}$ and ${ }^{240} \mathrm{Pu}$.

Despite these promising outcomes, the TDGCM+GOA approach in its current state still suffers from a major limitation: the presence of discontinuities in the collective landscape [13]. This problem is related to the lacuna of the many body Hilbert space explored by our approach. As a practical consequence, it prevents us from describing correctly the dynamics through scission, up to two well separated fragments. Although it is clear that an enrichment of the description space is required, there is no consensus on what physical ingredients should be added first. Adding more collective variables to our current description is a possible option. However, up to now no numerical tool was capable of computing a realistic TDGCM+GOA fission calculation within a $\mathrm{n}\left(i^{2}\right)$-dimensional collective space. A complementary alternative consists in the inclusion of single particle excitations into the many body wave function. This kind of approach attempted for instance in [21] requires building an extended formalism and has not yet been applied to a realistic calculation of fission dynamics. 


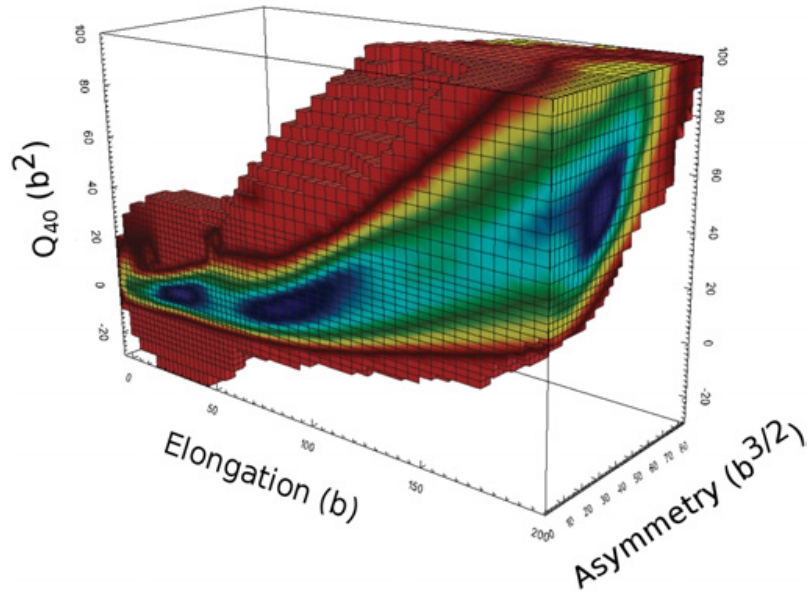

Figure 2. Preliminary 3-dimensional potential energy surface for ${ }^{240} \mathrm{Pu}$ in the collective space spanned by $\left(q_{20}, q_{30}, q_{40}\right)$.

\section{Toward the description of fission dynamics within a 3-dimensional collective space}

Although possible in principle, extending our current approach to $N>2$ collective variables rapidly increases the computational cost. For a regular discretization of a hyper-cubic collective domain, each additional dimension multiplies both the pre-calculation time of the potential energy surface and the evolution time of the TDGCM equations by approximately a factor 100 . To tackle this numerical challenge, we develop the code FELIX [22] aiming at solving the TDGCM+GOA equation in a collective space of arbitrary dimension. Since its first release under an open source GPL-2 license, continuous efforts have been spent to improve its scalability and performance. In its current version, FELIX relies first of all on the efficient spectral finite element discretization of the collective space. As a sub-category of finite elements, this approach enables handling irregular mesh on non hypercubic domains along with the possibility to apply two types of refinement (namely $h$ and p) [23]. The h-refinement consists in a spatial refinement of the mesh where such operation is most needed for the accuracy of the solution. The p-refinement strategy takes the party of increasing the degree of the polynomial basis used to represent the numerical solution. The use of both $\mathrm{h}$ - and p-refinement is a standard way to reach the exponential convergence property characteristic of the finite element method. In addition, the polynomial basis provided by the spectral element method is orthogonal up to a Gauss-LegendreLobatto quadrature approximation. This property makes possible an explicit resolution of the time integration of Eq. (1). In our implementation, this integration relies on a high order, unitary Krylov propagator which ensures its stability.

Leveraging the flexibility on the mesh, we estimate that a full 3-dimensional calculation would require of the order of $5.10^{6}$ points in the collective space. To test the feasibility of such a calculation, we generate the $3 \mathrm{D}$ potential energy surface of ${ }^{240} \mathrm{Pu}$ in the space spanned by the collective variables $\left(q_{20}, q_{30}, q_{40}\right)$ and up to $q_{20}=200 \mathrm{~b}$. The potential energy associated to this sub-space is plotted in Fig. 2. It roughly corresponds to one third of the total domain required. Based on this potential energy surface, we performed a first 3dimensional evolution of the system up to $10 \mathrm{zs}\left(10^{-21} \mathrm{~s}\right)$. This preliminary calculation, successfully converged within 135 cpu.h on an Intel(R) Core(TM) i7-4712MQ, enlightens the feasibility of 3-dimensional TDGCM+GOA with nowadays computational resources. Work is now in progress toward computing the first fission yields based on a 3-dimensional TDGCM+GOA framework.

\section{Conclusion}

In this work we use a fully quantum mechanical approach to compute fission fragment yields for neutron-induced fission with low energy neutrons. This approach provides robust qualitative results on describing the asymmetric fission of ${ }^{240} \mathrm{Pu}$ and ${ }^{236} \mathrm{U}$ within a 2-dimensional collective space. Thanks to the development of FELIX, the calculation of fission yields based on three collective coordinates seems reachable with current computational resources.

The research was carried out under the US-France International Agreement on Cooperation on Fundamental Research Supporting Stockpile Stewardship. This work was partly performed under the auspices of the U.S. Department of Energy by Lawrence Livermore National Laboratory under Contract DE-AC5207NA27344.

\section{References}

[1] M. Bernas, S. Czajkowski, P. Armbruster, H. Geissel, P. Dessagne, C. Donzaud, H.R. Faust, E. Hanelt, A. Heinz, M. Hesse et al., Physics Letters B 331, 19 (1994)

[2] K.H. Schmidt, S. Steinhäuser, C. Böckstiegel, A. Grewe, A. Heinz, A.R. Junghans, J. Benlliure, H.G. Clerc, M. de Jong, J. Müller et al., Nuclear Physics A 665, 221 (2000)

[3] M. Caamaño, O. Delaune, F. Farget, X. Derkx, K.H. Schmidt, L. Audouin, C.O. Bacri, G. Barreau, J. Benlliure, E. Casarejos et al., Phys. Rev. C 88, 024605 (2013)

[4] J.F. Martin, J. Taieb, A. Chatillon, G. Bélier, G. Boutoux, A. Ebran, T. Gorbinet, L. Grente, B. Laurent, E. Pellereau et al., Eur. Phys. J. A 51, 174 (2015)

[5] J.J. Griffin, J.A. Wheeler, Phys. Rev. 108, 311 (1957)

[6] P. Reinhard, K. Goeke, Rep. Prog. Phys. 50, 1 (1987)

[7] H. Goutte, J.F. Berger, P. Casoli, D. Gogny, Phys. Rev. C 71, 024316 (2005)

[8] W. Younes, D. Gogny, Lawrence Livermore National Laboratory, Tech. Rep. LLNL-TR-586678, 2012 (2012)

[9] W. Younes, D. Gogny, Lawrence Livermore National Laboratory, Tech. Rep. LLNL-TR-586694, 2012 (2012)

[10] D. Regnier, N. Dubray, N. Schunck, M. Verrière, Phys. Rev. C 93, 054611 (2016)

[11] P. Möller, D.G. Madland, A.J. Sierk, A. Iwamoto, Nature 409, 785 (2001)

[12] W. Younes, D. Gogny, Phys. Rev. C 80, 054313 (2009) 
[13] N. Dubray, D. Regnier, Computer Physics Communications 183, 2035 (2012)

[14] N. Schunck, D. Duke, H. Carr, A. Knoll, Phys. Rev. C 90, 054305 (2014)

[15] P. Schillebeeckx, C. Wagemans, A.J. Deruytter, R. Barthélémy, Nuclear Physics A 545, 623 (1992)

[16] K. Nishio, Y. Nakagome, I. Kanno, I. Kimura, J. Nucl. Sci. Technol. 32, 404 (1995)

[17] J. Bartel, P. Quentin, M. Brack, C. Guet, H.B. Håkansson, Nuclear Physics A 386, 79 (1982)

[18] J.F. Berger, M. Girod, D. Gogny, Nuclear Physics A 428, 23 (1984)
[19] J.F. Berger, M. Girod, D. Gogny, Computer Physics Communications 63, 365 (1991)

[20] C. Wagemans, The Nuclear Fission Process, 1st edn. (CRC Press, 1991), ISBN 0-8493-5434-X

[21] R. Bernard, H. Goutte, D. Gogny, W. Younes, Phys. Rev. C 84, 044308 (2011)

[22] D. Regnier, M. Verrière, N. Dubray, N. Schunck, Computer Physics Communications 200, 350 (2016)

[23] L.R. Ram-Mohan, Finite Element and Boundary Element Applications in Quantum Mechanics (Oxford University Press, 2002), ISBN: 978-0-19-852522-6 